\title{
Controlled release of diclofenac sodium from pH-responsive carrageenan-g-poly(acrylic acid) superabsorbent hydrogel
}

\author{
HOSSEIN HOSSEINZADEH \\ Department of Chemistry, University of Payame Noor, P.O. Box 59716-19411, West Azerbaijan, \\ Miandoab, Iran \\ e-mail: h_hoseinzadeh@pnu.ac.ir
}

MS received 16 December 2009; revised 13 March 2010; accepted 4 May 2010

\begin{abstract}
In this paper, controlled release of diclofenac sodium (DS) from $\mathrm{pH}$-sensitive carrageenan- $g$ poly(acrylic acid) superabsorbent hydrogels was investigated. The hydrogels were prepared by graft copolymerization of acrylic acid (AA) onto kappa-carrageenan, using ammonium persulfate (APS) as a free radical initiator in the presence of methylene bisacrylamide (MBA) as a crosslinker. Infrared spectroscopy was carried out to confirm the chemical structure of the hydrogel. Moreover, morphology of the samples was examined by scanning electron microscopy (SEM). The synthesized hydrogels were subjected to equilibrium swelling studies in simulated gastric and intestinal fluids (SGF and SIF). Hydrogels containing drug DS, at different drug-to-polymer ratios, were prepared by direct adsorption method. The loading yield was found to depend on both the impregnation time and the amount of encapsulated drug. In vitro drug-release studies in different buffer solutions showed that the most important parameter affecting the drug-release behaviour of hydrogels is the $\mathrm{pH}$ of the solution. The mechanism involved in release was Fickian $(n \leq 0.43, n=0.348)$ and Super Case II kinetics $(n>1, n=1.231)$ at pH 1.2 and 7.4, respectively.
\end{abstract}

Keywords. Carrageenan; hydrogel; acrylic acid; diclofenac sodium; controlled release.

\section{Introduction}

Superabsorbent polymers (SAPs) are special soft and pliable polymeric materials that can absorb large quantities of water, saline or physiological solutions while the absorbed solutions are not removable even under pressure. ${ }^{1,2}$ Because of their superior properties, they have found extensive applications such as disposable diapers, feminine napkins, drug delivery systems, and soil for agriculture and horticulture. ${ }^{3-5}$

Superabsorbent polymers have been widely studied since the first SAP was developed by the US Department of Agriculture in 1961. ${ }^{6}$

The properties of the swelling medium (e.g. $\mathrm{pH}$ and ionic strength) affect the swelling characteristics. SAPs responding to external stimuli such as heat, $\mathrm{pH}$, electric field, and chemical environments are often referred to as 'intelligent' or 'smart' polymers. Among these, $\mathrm{pH}$-sensitive hydrogels have been extensively investigated for potential use in site-specific delivery of drugs to specific regions of the gastrointestinal tract and have been prepared for delivery of low molecular weight drugs. Therefore, these hydrogels have important applications in the field of medicine, pharmacy, and biotechnology. ${ }^{4,5}$

There has been considerable interest in recent years in developing controlled or sustained drug delivery systems by using biopolymers. Controlled release technologies are used to deliver compounds like drugs, pesticides or fragrances at prescribed rates, together with improved efficacy, safety and convenience. Controlled drug release may be achieved using various devices, for example: mechanical pumps; osmotic pumps; diffusioncontrolled systems containing reservoirs or matrix systems; chemically-controlled systems composed of biodegradable or non-biodegradable polymers; swelling-controlled systems and magnetically controlled systems. ${ }^{7}$ The principal requirement of any controlled release system is that the release profile and rate are controlled. Controlled or sustained release drugs provide many advantages in comparison with conventional forms including reduced side effects, drug concentration kept at effective levels in 
plasma, improved utilization of drug and decreased dosing times. ${ }^{8}$

In this study, the utility of an anionic SAP prepared from graft copolymerization of acrylic acid onto kappa-carrageenan, for the controlled release of non-steroidal anti-inflammatory drug has been investigated. Drug absorption and release capacities of hydrogel systems and influence of $\mathrm{pH}$ of the medium on the release properties were also examined.

\section{Experimental}

\subsection{Materials}

The polysaccharide, kappa-carrageenan $(\mathrm{kC}, \mathrm{MW}=$ 100,000, from Condinson Co., Denmark), $N, N^{\prime}-$ methylene bisacrylamide (MBA, from Merck), and ammonium persulfate (APS, from Fluka) were of analytical grade and used without further purification. Acrylic acid (AA from Merck) as ionic monomer was used after vacuum distillation for removing inhibitor. Diclofenac sodium (DS) was obtained from Jaberebne Hayan Pharmaceutical Co. (Tehran, Iran). The chemical formula of diclofenac is shown in figure 1. Double distilled water was used for the hydrogel preparation and swelling measurements.

\subsection{Preparation of hydrogel}

Certain amounts of distilled water $(30 \mathrm{~mL})$ and $\mathrm{kC}$ $(1.0 \mathrm{~g})$, were added to a three-neck reactor equipped with a mechanical stirrer (RZR 2021, a three-blade propeller type, Heidolph, Schwabach, Germany), while stirring $(350 \mathrm{rpm})$. The reactor was placed in a thermostated water bath preset at $80^{\circ} \mathrm{C}$ for $20 \mathrm{~min}$. After dissolving $\mathrm{kC}$ and homogenizing the mixture, the initiator $\left(0.01-0.40 \mathrm{~g}\right.$ APS in $\left.5 \mathrm{~mL} \mathrm{H}_{2} \mathrm{O}\right)$ and methylene bisacrylamide $\left(0.05-0.20 \mathrm{~g}\right.$ in $\left.5 \mathrm{~mL} \mathrm{H}_{2} \mathrm{O}\right)$ were simultaneously added and the reaction mixture

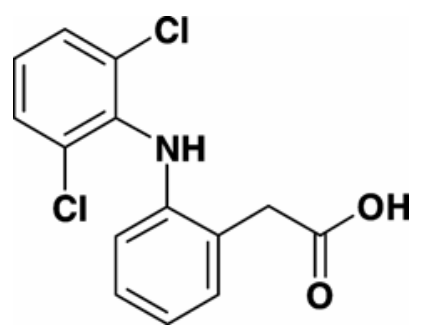

Figure 1. Chemical structure of diclofenac. was stirred for $20 \mathrm{~min}$. Then, the monomer (AA, $2 \cdot 0-8 \cdot 0 \mathrm{~g}$ ) was added and gelation was observed after $30 \mathrm{~min}$. After $1 \mathrm{~h}$, the mixture was treated with $1 \mathrm{~N}$ sodium hydroxide for $70 \%$ neutralization of the carboxylic groups of the grafted poly(acrylic acid). Finally, the gel product was poured into $100 \mathrm{~mL}$ of ethanol for $2 \mathrm{~h}$ and then scissored to small pieces. The non-solvent ethanol was then decanted and $100 \mathrm{ml}$ fresh ethanol was added. The particles were allowed for $24 \mathrm{~h}$ to completely solidify. The dewatered gel particles were filtered and dried in oven at $45^{\circ} \mathrm{C}$ for $6 \mathrm{~h}$. After grinding, the powdered superabsorbent hydrogel was stored away from moisture, heat and light.

\subsection{Swelling measurements}

An accurately weighed sample of the powdered superabsorbent $(0.2 \pm 0.001 \mathrm{~g})$ with average particle sizes between 40 and 60 mesh $(250$ and $350 \mu \mathrm{m})$ was immersed in distilled water $(200 \mathrm{~mL})$ and allowed to soak for $3 \mathrm{~h}$ at room temperature. The equilibrium swelling (ES) capacity was measured twice at room temperature according to a conventional tea bag method and using the following formula:

$$
\begin{aligned}
& E S(g / g)= \\
& \frac{\text { Weight of swollen gel - Weight of dried gel }}{\text { Weight of dried gel }}
\end{aligned}
$$

\subsection{Loading of drug}

Powdered samples ( $1 \pm 0.0001 \mathrm{~g})$, with average particle sizes between 40 and 60 mesh $(250-350 \mu \mathrm{g})$, were accurately weighted and immersed in an alkaline solution of DS $(2.0 \mathrm{~g}$ dissolved in $230 \mathrm{~mL}$ distilled water) at $0^{\circ} \mathrm{C}$ for $25 \mathrm{~h}$. The swollen hydrogels loaded with drug were placed in a vacuum oven and dried under vacuum at $37^{\circ} \mathrm{C}$.

\subsection{Determination of loading efficiency}

The amount of drug content entrapped in the hydrogels was determined by an indirect method. After the gel preparation, the washings were collected, filtered with a $0.45 \mu \mathrm{m}$ Millipore filter and tested at $\lambda_{\max } 276 \mathrm{~nm}$ using UV/VIS spectrophotometer (UV1201, Shimadzu, Kyoto, Japan). 
The drugs entrapped exhibited the same $\lambda_{\max }$ as the free drug. This clearly indicates that the drugs entrapped have not undergone any possible chemical reaction during the matrix formation. The difference between the amount of drug initially employed and the drug content in the washings is taken as an indication of the amount of drug entrapped:

\section{Drug entrapment $(\%)=$}

$$
\frac{\text { Mass of drug present in hydrogel }}{\text { Theoretical mass of DS }} \times 100
$$

\subsection{In vitro drug release}

The in vitro release of DS was evaluated using dissolution methodology in simulated gastric and intestinal fluids (SGF and SIF). In vitro release was carried out in duplicate by incubating $0 \cdot 1 \pm 0.0001 \mathrm{~g}$ of the DS-loaded hydrogels into a cellophane membrane dialysis bag (D9402, SIGMA-ALDRICH) in $50 \mathrm{~mL}$ of buffer solution (either $\mathrm{pH} 1.2$ or 7.4 ) at $37^{\circ} \mathrm{C}$. At specific time intervals, $1 \mathrm{~mL}$ aliquots of sample was withdrawn through a sampling syringe attached with a $0.45 \mu \mathrm{m}$ Millipore filter and after suitable dilution, the concentration of released drug was measured by UV spectrophotometer at $276 \mathrm{~nm}$. The drug release percent was calculated twice using the following equation:

$$
\text { Released drug }(\%)=\frac{R_{t}}{L} \times 100 \text {, }
$$

where $L$ and $R_{t}$ represent the initial amount of drug loaded and the final amount of drug released at time $t$.

\subsection{Instrumental analysis}

Fourier transform infrared (FTIR) spectra of samples were taken in $\mathrm{KBr}$ pellets, using an $\mathrm{ABB}$ Bomem MB-100 FTIR spectrophotometer (Quebec, Canada), at room temperature. The surface morphology of the gel was examined using scanning electron microscopy (SEM). After Soxhlet extraction with methanol for $24 \mathrm{~h}$ and drying in an oven, superabsorbent powder was coated with a thin layer of gold and imaged in a SEM instrument (Leo, 1455 VP).

\section{Results and discussion}

\subsection{Synthesis and spectral characterization}

Scheme 1 shows a simple structural proposal of the graft copolymerization of AA monomers onto the $\mathrm{kC}$ backbones and crosslinking of the resulted graft copolymer. In the first step, the thermally dissociating initiator, i.e. APS, is decomposed under heating $\left(80^{\circ} \mathrm{C}\right)$ to produce sulfate anion-radicals. Then the anion-radicals abstract hydrogen from the $\mathrm{kC}$ backbones to form corresponding macroinitiators. These macroradicals initiate grafting of AA onto $\mathrm{kC}$ backbones leading to a graft copolymer. Crosslinking reaction also occurred in the presence of the crosslinker, i.e. MBA.

The grafting was confirmed by comparing the FTIR spectra of the polysaccharide substrate with that of the grafted products. Figure 2 shows the FTIR spectra of non-modified $\mathrm{kC}$ and $\mathrm{kC}-g$ poly(AA) hydrogel. The bands observed at 848,919 , 1022 and $1222 \mathrm{~cm}^{-1}$ can be attributed to D-galactose-4-sulfate, 3,6-anhydro-D-galactose, glycosidic linkage and ester sulfate stretching of $\mathrm{kC}$, respectively (figure 2a). The broad band at $3200-3400 \mathrm{~cm}^{-1}$ is due to stretching of $-\mathrm{OH}$ groups of substrate. The superabsorbent hydrogel product comprises a $\mathrm{kC}$ backbone with side chains that carry carboxylic and carboxylate functional groups that are evidenced by peaks at 1718,1557 and $1409 \mathrm{~cm}^{-1}$ respectively. The very intense characteristic band at $1557 \mathrm{~cm}^{-1}$ is due to $\mathrm{C}=\mathrm{O}$ asymmetric stretching in carboxylate anion that is reconfirmed by another sharp peak at $1409 \mathrm{~cm}^{-1}$ which is related to the symmetric stretching mode of the carboxylate anion. The stretching band of $\mathrm{C}=\mathrm{O}$ in carboxamide functional groups of the MBA portion of the hydrogel overlapped with the $\mathrm{C}=\mathrm{O}$ asymmetric stretching of carboxylate anion at $1557 \mathrm{~cm}^{-1}$.

To obtain additional evidence of grafting, a similar polymerization was conducted in the absence of the crosslinker. After extracting the homopolymer and unreacted monomers using a cellophane membrane dialysis bag, an appreciable amount of grafted $\mathrm{kC}(89 \%)$ was observed. The graft copolymer spectrum was very similar to figure $2 \mathrm{~b}$. Also according to preliminary measurements, the sol (soluble) content of the hydrogel networks was as little as $2.4 \%$. This fact practically proves that all monomers are almost involved in the polymer network. So, the monomers percent in the network will be very similar to that of the initial feed of reaction. 


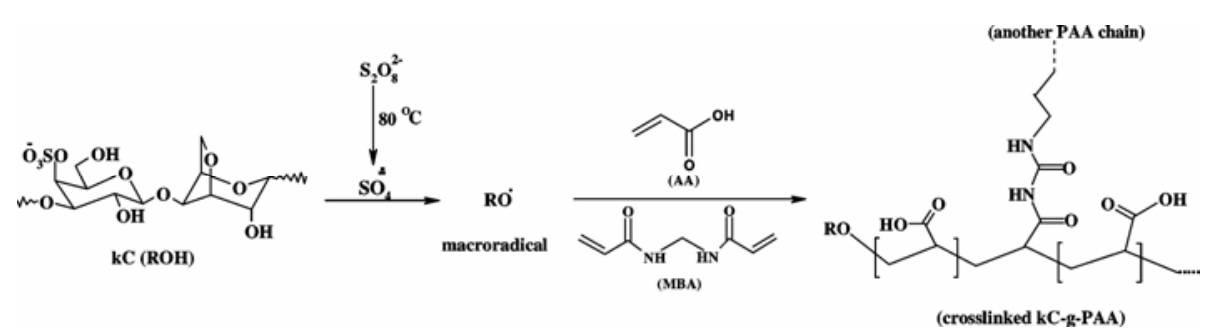

Scheme 1. Proposed mechanistic pathway for synthesis of kC-g-poly(acrylic acid) hydrogel.

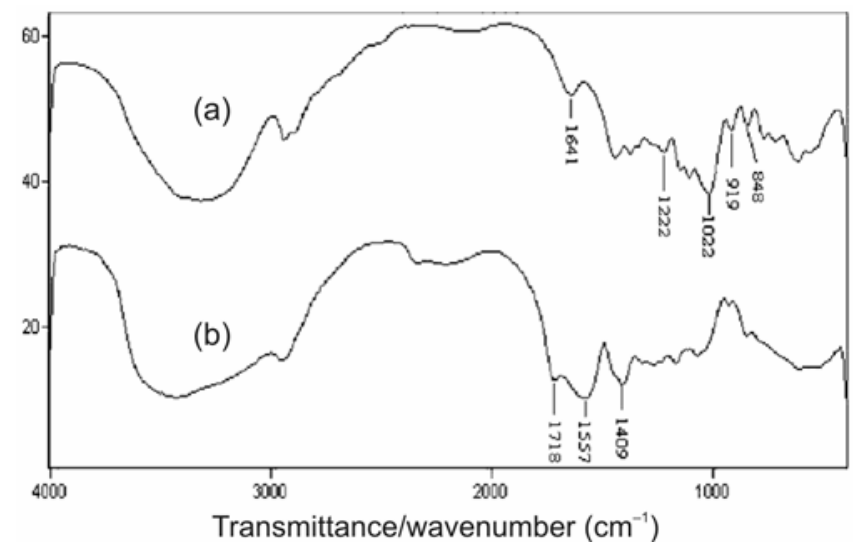

Figure 2. FTIR spectra of $\mathrm{kC} \mathrm{(a)} \mathrm{and} \mathrm{kC-g-poly(acrylic}$ acid) hydrogel (b).

\subsection{Scanning electron microscopy}

One of the most important properties that must be considered is hydrogel microstructure morphologies. The surface morphology of the samples was investigated by scanning electron microscopy (SEM). Figure 3 shows an SEM micrograph of the polymeric hydrogels obtained from the fracture surface. The hydrogel has a porous structure. It is supposed that these pores are the regions of water permeation and interaction sites of external stimuli with the hydrophilic groups of the graft copolymers.

\subsection{Optimization of the grafting variables}

In this work, the main factors affecting on the grafting conditions, i.e. concentration of MBA, AA, APS, and $\mathrm{kC}$ were systematically optimized to achieve superabsorbent with maximum water absorbency.

\section{3a Effect of MBA concentration: Crosslinks} have to be present in a hydrogel in order to prevent dissolution of the hydrophilic polymer chains in an aqueous environment. The crosslinked nature of hydrogels makes them insoluble in water. The efficiency of the incorporated crosslinker controls the overall crosslink density in the final hydrogel. The effect of crosslinker concentration on swelling capacity of kC-g-poly(AA) was investigated. As shown in figure 4, more values of absorbency are obtained by lower MBA concentration as reported by pioneering scientists. ${ }^{9}$ In fact, higher crosslinker concentrations decrease the free space between the copolymer chains. Therefore, the resulted highly crosslinked rigid structure can not be expanded and hold a large quantity of water. The maximum absorbency $(351 \mathrm{~g} / \mathrm{g})$ is achieved at $0.00194 \mathrm{~mol} / \mathrm{L}$ of MBA.

$3.3 \mathrm{~b}$ Effect of AA concentration: The effect of monomer concentration on swelling capacity of the hydrogel was studied by varying the AA concentration from 0.47 to $1.90 \mathrm{~mol} / \mathrm{L}$ (figure 5). Enhanced monomer concentration increases the diffusion of AA molecules into the $\mathrm{kC}$ backbone that consequently causes an increase in water absorbency. In addition, higher AA content enhanced the hydrophilicity of the hydrogel. The swelling-loss after the maximum may be originated from the increased chance of chain transfer to AA molecules and increase in viscosity of the reaction which restricts the movement of the reactants.

To obtain an additional evidence of grafting (or swelling) dependency to the monomer concentration, the percentage of grafting efficiency (\%Ge) was evaluated with the following weight-basis equation as reported by Fanta: ${ }^{10}$

$$
\% \mathrm{Ge}=\frac{\text { PAA grafted }}{\text { Monomer charged }} \times 100 \text {. }
$$

The \%Ge stands for the grafted PAA formed from initial monomer charged. The $\% \mathrm{Ge}$ parameter was 
found to be increased (75-89\%) by enhancement of acrylic acid concentration from 0.47 to $1.52 \mathrm{~mol} / \mathrm{L}$ and then, it is decreased.

3.3c Effect of APS concentration: The relationship between the initiator concentration and water absorbency values was studied by varying the APS concentration from 0.0065 to $0.052 \mathrm{~mol} / \mathrm{L}$ (figure 6). It is observed that the absorbency is substantially increased with increasing in the APS concentration and then it is decreased. Initial increment in water absorbency may be attributed to increased number of active free radicals on the carrageenan backbone. Subsequent decrease in swelling is originated from an increase in terminating step reaction via bimolecular collision which, in turn, causes to enhance

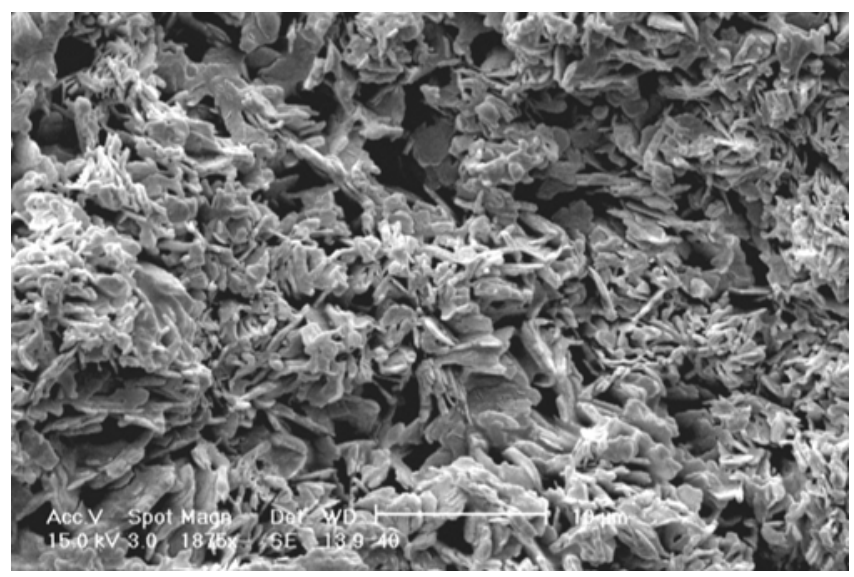

Figure 3. SEM photograph of the hydrogel. Surfaces were taken at a magnification of 1000, and the scale bar is $10 \mu \mathrm{m}$.

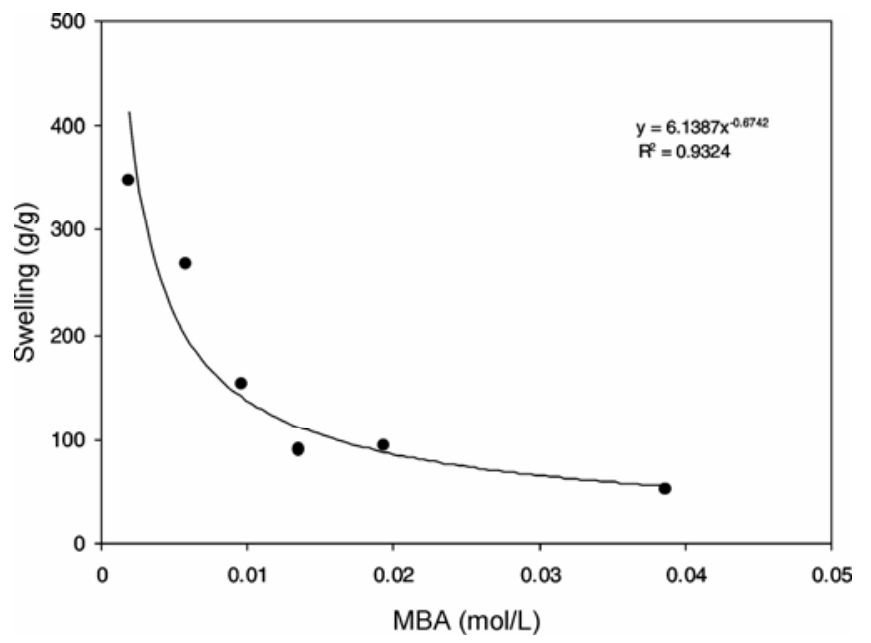

Figure 4. Effect of crosslinker concentration on swelling capacity. crosslinking density. This possible phenomenon is referred to as 'self-crosslinking' by Chen and Zhao. ${ }^{11}$ In addition, the free radical degradation of $\mathrm{kC}$ backbones by sulfate radical-anions is an additional reason for swelling-loss at higher APS concentration. The proposed mechanism for this possibility is reported in the previous work. ${ }^{12} \mathrm{~A}$ similar observation is reported by Hsu et al in the case of degradation of chitosan with potassium persulfate. $^{13}$

3.3d Effect of polysaccharide concentration: The swelling dependency on $\mathrm{kC}$ amount is shown in figure 7. Hydrogel swelling linearly increased with increasing $\mathrm{kC}$ from 1.0 to $4.0 \mathrm{wt} \%$ from 77 to

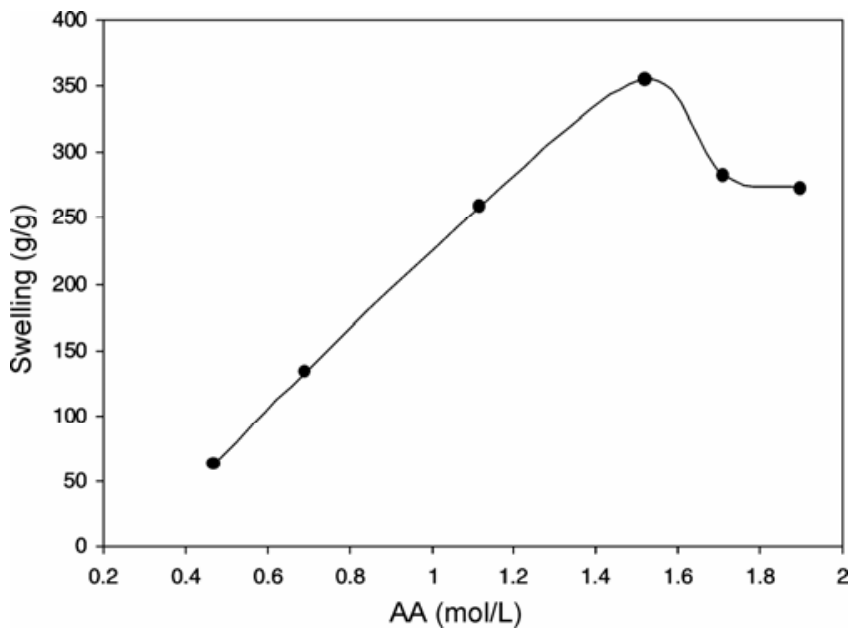

Figure 5. Effect of monomer concentration on swelling capacity.

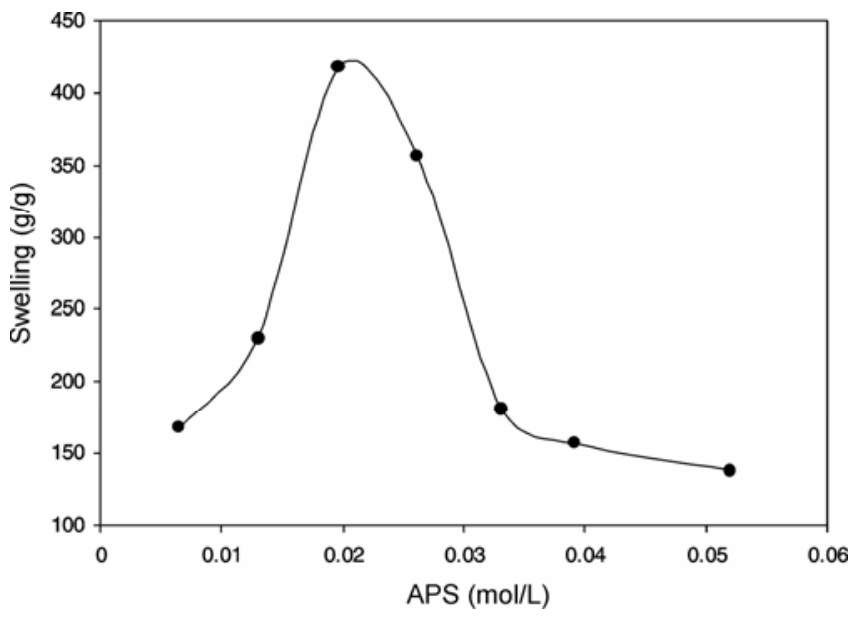

Figure 6. Effect of initiator concentration on swelling capacity. 
$477 \mathrm{~g} / \mathrm{g}$, respectively. This behaviour is attributed to the increased grafted $\mathrm{kC}$ sites. However, on further increase in the substrate concentration, the reaction medium viscosity restricts the movement of the macroradicals.

\subsection{Equilibrium swelling at various $p H$ solutions}

Ionic superabsorbent hydrogels exhibit swelling changes at a wide range of pHs. Therefore, in this series of experiments, equilibrium swelling for the synthesized hydrogels was measured in different $\mathrm{pH}$ solutions ranged from 1.0 to 12.0 (figure 8 ). Since the swelling capacity of all 'anionic' hydrogels is appreciably decreased by addition of counter ions (cations) to the swelling medium, no buffer solu-

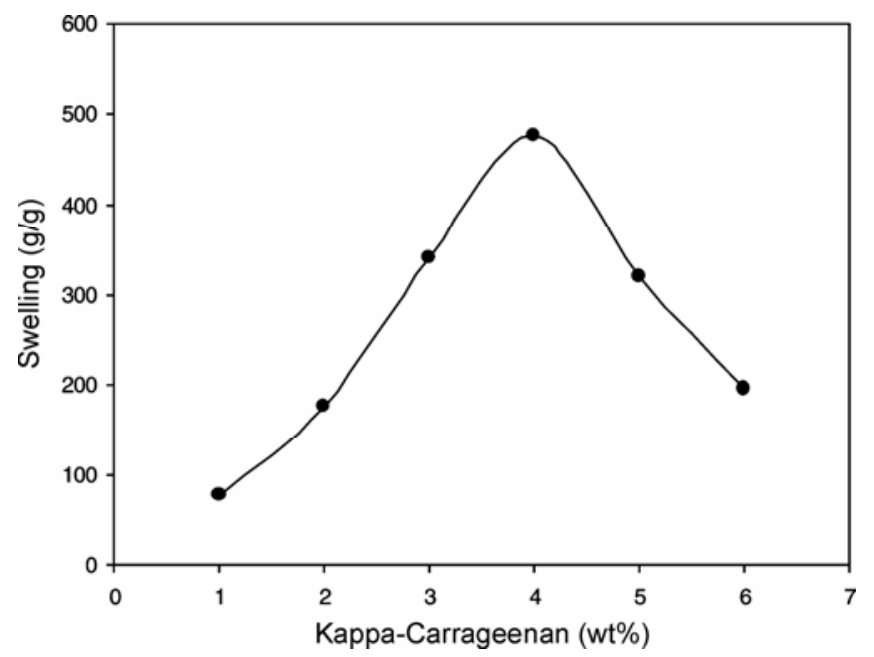

Figure 7. Effect of $\mathrm{kC}$ amount on swelling capacity.

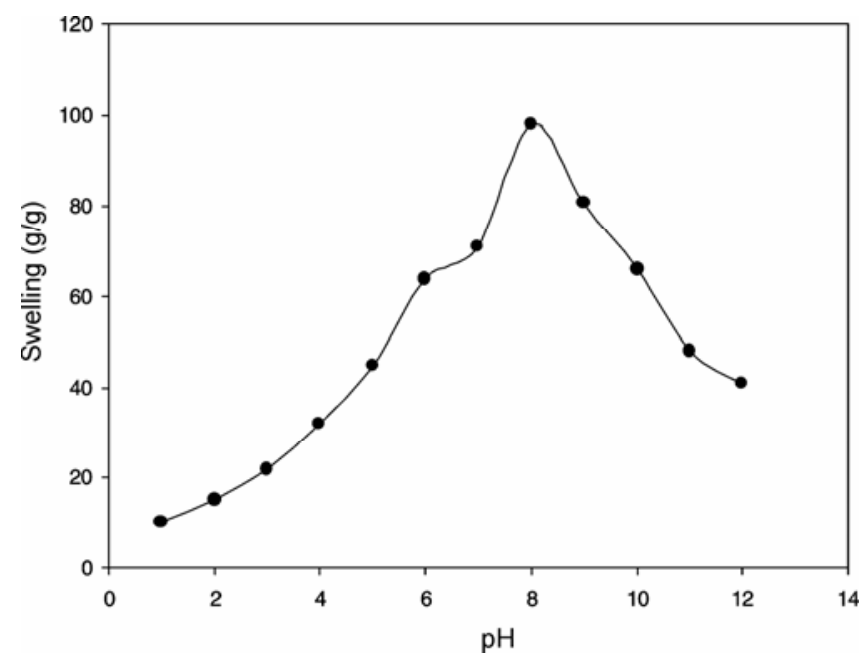

Figure 8. Effect of $\mathrm{pH}$ of solution on swelling of $\mathrm{kC}-\mathrm{g}$ poly(acrylic acid) hydrogel. tions were used. Therefore, stock $\mathrm{NaOH}(\mathrm{pH} 12 \cdot 0)$ and $\mathrm{HCl}(1 \cdot 0)$ solutions were diluted with distilled water to reach desired basic and acidic $\mathrm{pHs}$, respectively. Maximum swelling $(98 \mathrm{~g} / \mathrm{g})$ was obtained at $\mathrm{pH} 8$. Under acidic pHs $(\leq 4)$, most of the carboxylate anions are protonated, so the main anion-anion repulsive forces are eliminated and consequently swelling values are decreased. However, some sort of attractive interactions ( $\mathrm{H}-\mathrm{O}$ hydrogen bonding) lead to decreased absorbencies. At higher $\mathrm{pHs}$ (4-8), some of carboxylate groups are ionized and the electrostatic repulsion between $\mathrm{COO}^{-}$groups causes an enhancement of the swelling capacity. The reason of the swelling-loss for the highly basic solutions $(\mathrm{pH}>8)$ is 'charge screening effect' of excess $\mathrm{Na}^{+}$ in the swelling media, which shields the solfunate and carboxylate anions and prevents effective anion-anion repulsion. Similar swelling-pH dependencies have been reported in the case of other hydrogel systems. ${ }^{14-16}$

\section{5 pH-responsiveness behaviour of $k C-g$ - poly (AA) hydrogel}

Since the synthesized hydrogel, kC-g-poly(AA), shows different swelling behaviours in acidic and basic $\mathrm{pH}$ solutions, we investigated the reversible swelling-deswelling behaviour of this hydrogel in solutions with $\mathrm{pH} \mathrm{2.0}$ and 9.0 (figure 9). At $\mathrm{pH} 9.0$, the hydrogel swells due to anion-anion repulsive electrostatic forces, while at $\mathrm{pH} 2 \cdot 0$, it shrinks within a few minutes due to protonation of the sol-funate and carboxylate anions. This swelling-

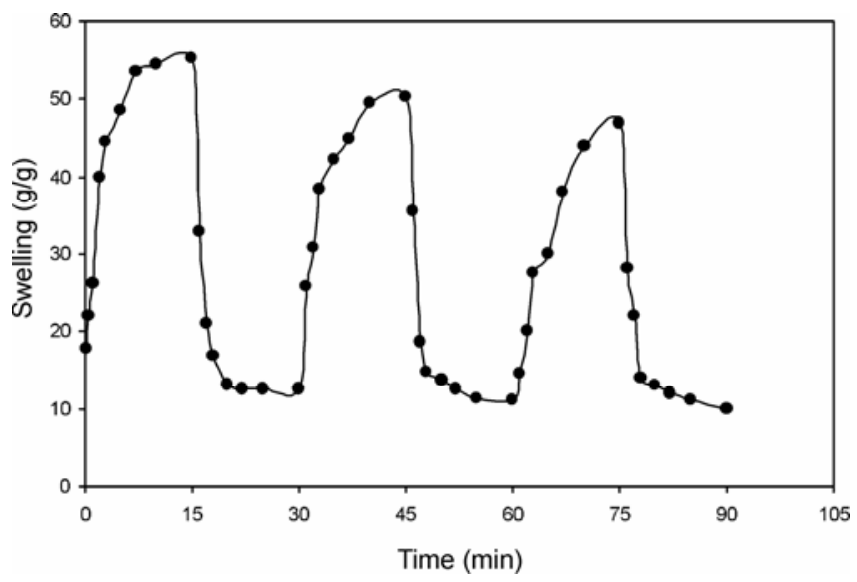

Figure 9. On-off switching behaviour as reversible pulsatile swelling $(\mathrm{pH} 8.0)$ and deswelling $(\mathrm{pH} 2.0)$ of the $\mathrm{kC}$ - $g$-poly(acrylic acid) hydrogel. The time interval between the $\mathrm{pH}$ changes was $15 \mathrm{~min}$. 
deswelling behaviour of the hydrogels makes them as suitable candidate for designing drug delivery systems. Such on-off switching behaviour as reversible swelling and deswelling has been reported for other ionic hydrogels. ${ }^{17-19}$

\subsection{Effect of drug concentration on loading efficiency}

The effect of the initial concentration of diclofenac sodium solution on the adsorption capacities of hydrogels is shown in figure 10. As can be seen from the figure, an increase in drug concentration in the swelling medium increased the amount of adsorbed drug, as observed in many adsorption studies. $^{20-22}$ This result can also be obtained from the following equation:

$$
q_{e}=\left(\frac{\left(C_{i}-C\right)}{m}\right) \times V_{t}
$$

where $q_{e}$ is in mg adsorbate per gram of dry adsorbent, $C_{i}$ and $C$ are the initial and equilibrium concentrations of adsorbate solution in $\mathrm{mg} / \mathrm{ml}, V_{t}$ is the volume of solution treated in $\mathrm{ml}$, and $m$ is the mass of dry adsorbent, in g. As can be seen from (5), an increase in concentration of drug in the gel system increased $q_{e}$ values.

\subsection{Effect of loading time on loading efficiency}

The amounts of the loaded drug in superabsorbent hydrogels was significantly affected by the impreg-

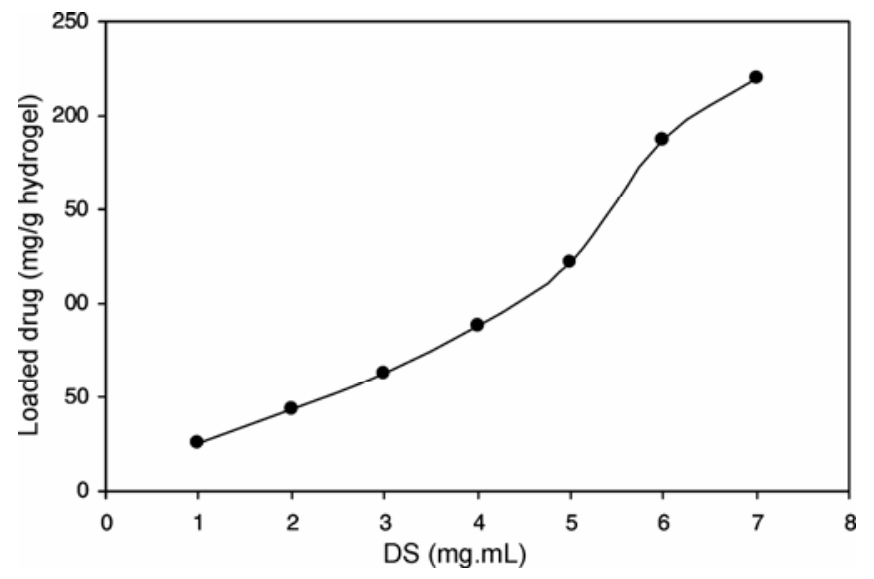

Figure 10. Effect of drug concentration on the adsorption capacities of hydrogel. nation times (figure 11). It is obvious that with increasing the loading time, the amount of drug loaded is initially increased and then begins to level-off. The initial increment in the amounts of the loaded drug with increasing the loading time can be ascribed to the increased drug diffusion into the swollen matrix. The most efficient time of loading efficiency was $7 \mathrm{~h}$, where a major amount of drug was encapsulated.

\subsection{In vitro release behaviour of hydrogels}

To determine the potential application of $\mathrm{kC}$-based superabsorbent containing a pharmaceutically active compound, we have investigated the drug release behaviour form this system under physiological conditions. The percent of released drug from the polymeric carriers as a function of time is shown in figure 12. The concentration of DS released at selected time intervals was determined by UV spectrophotometer. The DS-loaded hydrogels with high degrees of drug loading $(>80 \%)$ were prepared by the swelling-diffusion method. The amount of DS released in a specified time from the $\mathrm{kC}$-based hydrogel decreased as the $\mathrm{pH}$ of the dissolution medium was lowered (figure 12). At low $\mathrm{pH}$ values, electrostatic repulsion between the carboxylic acid groups of backbone is low, thus decreases gel swelling and minimizes release of DS via diffusion. However, in alkaline media the presence of $\mathrm{OH}^{-}$ increases the electrostatic repulsion between carboxylate groups, thus increases the gels swelling degree and so the release of DS increased. ${ }^{23,24}$

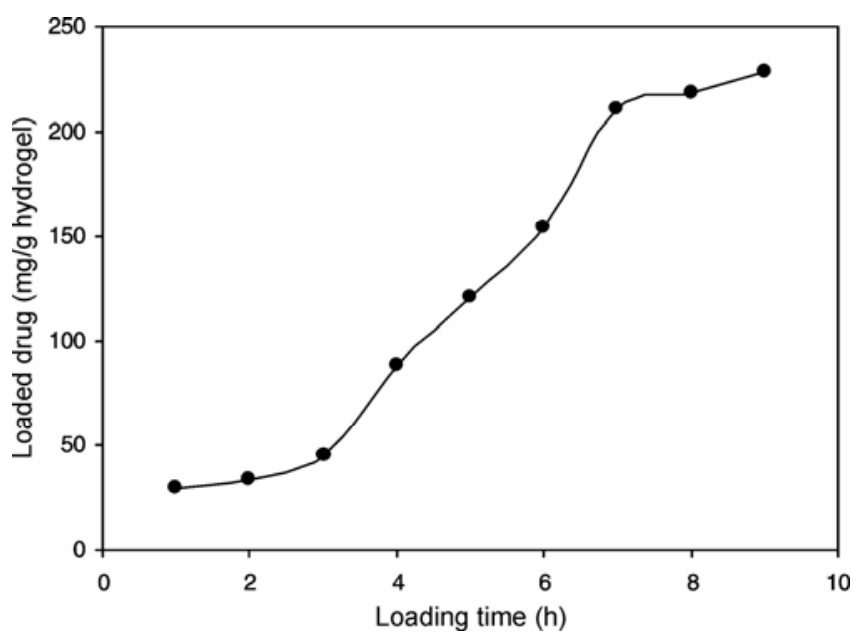

Figure 11. The dependency of the drug loading amount to the loading time. 


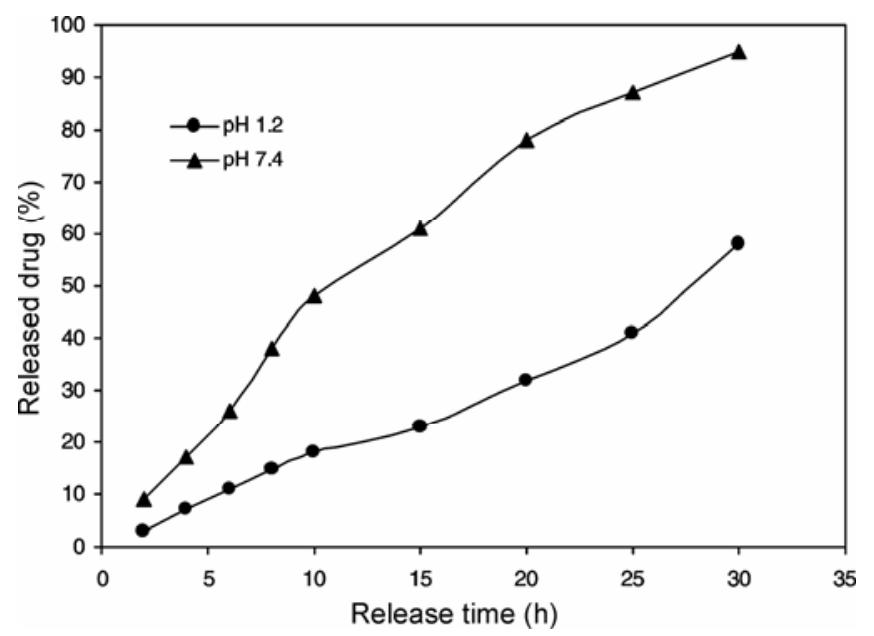

Figure 12. Release of DS from hydrogel carrier as a function of time and $\mathrm{pH}$ at $37^{\circ} \mathrm{C}$.

\subsection{Mechanism and mathematical modelling for drug release}

The mechanism of drug release from hydrogels is determined by different physical-chemical phenomena. According to Nixon, ${ }^{25}$ three steps lead to drug release from hydrogels in aqueous medium: (i) imbibition of the release medium into hydrogels, (ii) dissolution of the drug inside hydrogels and (iii) drug release by a diffusion process into the aqueous medium. The Korsmeyer-Peppas model (6), correlating drug release to time by a simple exponential equation for the fraction of release drug $<0.6$, has been used to evaluate drug release from controlled release polymeric devices, especially when the drug release mechanism is unknown or when there are more than one release mechanism. ${ }^{26,27}$

$$
\frac{M_{t}}{M_{\infty}}=k \cdot t^{n} .
$$

$M_{\mathrm{t}} / M_{\infty}$ is the proportion of drug released at time $t, k$ is the kinetic constant and the exponent $n$ has been proposed as indicative of the release mechanism. In this context, $n \leq 0.43$ indicates Fickian release and $n \quad 0.85$ indicates a purely relaxation controlled delivery which is referred as Case II transport. Intermediate values $0.43<n<0.85$ indicate an anomalous behaviour (non-Fickian kinetics corresponding to coupled diffusion/polymer relaxation). ${ }^{28,29}$ Occasionally, values of $n>1$ has been observed, which has been regarded as Super Case II kinetics. ${ }^{30,31}$ With the linear form of (6), plotting of $\ln M_{t} / M_{\infty}$ against $\ln t$, yielded the diffusion exponential $(n)$, the Pearson coefficient $\left(r^{2}\right)$ and the diffusion constant $(k)$. The results are summarized in table 1 .
Table 1. Analysis of release data from $\mathrm{kC}$-based hydrogels.

\begin{tabular}{lccc}
\hline $\mathrm{pH}$ & $n$ & $k\left(\mathrm{~min}^{-n}\right)$ & $r^{2}$ \\
\hline 1.2 & 0.348 & 1.3848 & 0.9782 \\
7.4 & 1.231 & 1.1823 & 0.9986 \\
\hline
\end{tabular}

Inspection of the results shown in table 1 indicates that the release of diclofenac sodium from hydrogels in $\mathrm{pH} 1.2$ presented an $n$ value of 0.348 indicative of a Fickian behaviour. The non-Fickian kinetics corresponds to the coupling of diffusion and polymer relaxation. On the other hand, the controlled release of drug from hydrogels in $\mathrm{pH} 7.4$ presented an $n$ value of 1.231 , indicating that the mechanism for release was included diffusion, swelling, relaxation and erosion process. The $k$ values were compared with the kinetics of diclofenac sodium release; in both medium the values were similar.

\section{Conclusion}

A novel biopolymer-based superabsorbent hydrogel was synthesized via graft copolymerization of acrylic acid (AA) onto kappa-carrageenan backbones in an aqueous solution using a persulfate initiator and a hydrophilic crosslinker. The study of FTIR spectra provides the graft copolymerization. The optimum reaction conditions to obtain maximum water absorbency $(347 \mathrm{~g} / \mathrm{g})$ were found to be: MBA $0.006 \mathrm{~mol} / \mathrm{L}$, AA $0.68 \mathrm{~mol} / \mathrm{L}$, APS $0.10 \mathrm{~mol} / \mathrm{L}$, and $\mathrm{kC} 4 \mathrm{wt} \%$.

The superabsorbent hydrogels exhibited high sensitivity to $\mathrm{pH}$, so that, several swelling changes of the hydrogel were observed with variations over a wide range ( $\mathrm{pH}$ 1-12). The reversible swellingdeswelling behaviour in solutions with acidic and basic $\mathrm{pH}$, contributes to the suitability of these hydrogels as candidates for controlled drug delivery systems. The loading and release of DS from the $\mathrm{pH}$-sensitive hydrogels was effective. The release value of $\mathrm{DS}$ from hydrogels at $\mathrm{pH} 7.4$ was higher than that at $\mathrm{pH} 1.2$ due to the electrostatic repulsion between carboxylate groups. The mechanism implicated in this process was Fickian and Super Case II kinetics.

The SAP delivery hydrogels presented in this study may serve as a platform for a wide range of pharmaceutical uses to improve the bioavailability of non-steroidal anti inflammatory drugs. 


\section{Acknowledgements}

This research work was supported by Bonyade Melli Nokhbegan of Iran and University Grant Programs.

\section{References}

1. Buchholz F L and Graham A T 1997 Modern superabsorbent polymer technology (New York: Wiley)

2. Peppa L B and Harland R S 1990 Absorbent polymer technology (Amsterdam: Elsevier)

3. Po R 1994 J. Macromol. Sci-Rev. Macromol. Chem. Phys. C34 607

4. Peppas N A and Mikes A G 1986 Hydrogels in medicine and pharmacy (Florida: CRC Press, Boca Raton)

5. Kost J 1995 Encyclopedia of controlled drug delivery (New York: Wiley)

6. United States Department of Agriculture 1961 UA Patent 3981100

7. Silver F and Doillon C 1989 Polymers, biocompatibility. In Interactions of biological and implantable materials (New York: VCH Publishers)

8. Desai K G H and Park H J 2005 J. Microencapsul. 22 377

9. Fanta G F 1996 Polymeric materials encyclopedia (Boca Raton, FL: CRC Press)

10. Fanta G F 1973 Block and graft copolymerization (Wiley: London)

11. Chen J and Zhao Y $2000 \mathrm{~J}$. Appl. Polym. Sci. 75808

12. Hosseinzadeh H, Pourjavadi A, Zohouriaan-Mehr M $\mathrm{J}$ and Mahdavinia G R $2005 \mathrm{~J}$. Bioact. Compat. Polym. 20475

13. Hsu S C, Don TM and Chiu W Y 2002 Polym. Degrad. Stab. 7573
14. Pourjavadi A, Sadeghi M and Hosseinzadeh H 2004 Polym. Adv. Technol. 15645

15. Park S E, Nho Y C, Lim Y M and Kim H 2004 J. Appl. Polym. Sci. 91636

16. Lu S, Duan M and Lin S 2001 J. Appl. Polym. Sci. 79 1665

17. Mahdavinia G R, Pourjavadi A and Zohuriaan-Mehr M J 2004 Polym. Adv. Technol. 15173

18. Ju H K, Kim S Y and Lee Y M 2001 Polymer 42 6851

19. Gan L H, Deen G R, Gan Y Y and Tam K C 2001 Eur. Polym. J. 371473

20. Sen M and Yakar A 2001 Int. J. Pharm. 22833

21. Akkas P, Sari M, Sen M and Guven O 1999 Radiat. Phys. Chem. 55717

22. Saraydin D, Karadag E, Oztop H N and Guven O 1994 Biomaterials 15917

23. Mahkam M, Doostie L and Siadat S O R 2006 Inflammopharmacology 1472

24. Mahkam M and Allahverdipoor M 2004 Drug Target. 12151

25. Nixon J R 1983 Release characterization of microcapsules. In Biomedical applications of microcapsulation (Boca Raton, FL: CRC Press)

26. Costa P and Lobo J M 2001 Europ. J. Pharm. Sci. 13 123

27. Korsmeyer R W, Gurny R and Doelker E 1983 Int. J. Pharm. 1525

28. Ritger P L and Peppas N A 1987 J. Control. Release 523

29. Ritger P L and Peppas N A 1987 J. Control. Release 537

30. Munday D L and Cox P L 2000 Int. J. Pharm. 12 179

31. Ranga R, Devi K D and Buri P 1988 Drug Dev. Ind. Pharm. 142299 